\title{
Optimization of Long-Run Average-Flow Cost in Networks With Time-Varying Unknown Demand
}

\author{
Dario Bauso, Franco Blanchini, and Raffaele Pesenti
}

\begin{abstract}
We consider continuous-time robust network flows with capacity constraints and unknown but bounded time-varying demand. The problem of interest is to design a control strategy off-line with no knowledge of the demand realization. Such a control strategy regulates the flow on-line as a function of the realized demand.

We address both the case of systems without and with buffers. The main novelty in this work is that we consider a convex cost which is a function of the long-run average-flow and average-demand. We distinguish a worst-case scenario where the demand is the worst-one from a deterministic scenario where the demand has a neutral behavior. The resulting strategies are called min-max or deterministically optimal respectively. The main contribution are constructive methods to design either min-max or deterministically optimal strategies. We prove that while the min-max optimal strategy is memoryless, i.e., it is a piece-wise affine function of the current demand, deterministically optimal strategy must keep memory of the average flow up to the current time.
\end{abstract}

Index Terms-Average flow cost, flow control, gradient-based control, min-max optimality, uncertain demand.

\section{INTRODUCTION}

W E frame this work within the several recent attempts to apply the tools of robust optimization to network flows [1], [2], [10], [11], [18], [23], [24].

Network flows describe flows of materials between different production/distribution sites (see, e.g., [25]). The problem is to design a strategy that returns the controlled flow as a function of the uncertain and time-varying demand.

Robust optimization is a relatively recent technique that describes uncertainty via sets and optimizes the worst-case cost over those sets (see, e.g., the introduction to the special issue [6]). Generally speaking, robust optimization aims at achieving the best cost under the worst uncertainty conditions. Some of the existing works (in particular [2], [11]) are centered around the idea of "adjusting" some of the variables to the outcome of the uncertainty. In other words some variables are decided before the uncertainty realization while the rest are decided after the uncertainty realization. Such a problem formulation is known under different names such as "Adjustable Robust

Manuscript received September 29, 2007; revised October 20, 2008. First published November 24, 2009; current version published January 13, 2010. This work was supported in part by MURST-PRIN Grants 2006090243 and 2007ZMZK5T. Recommended by Associate Editor P. A. Parrilo.

D. Bauso is with the Dip. di Ingegneria Informatica, Università di Palermo, Palermo I-90128, Italy (e-mail: dario.bauso@unipa.it).

F. Blanchini is with the Dip. di Matematica e Informatica, Università di Udine, Udine 33100, Italy (e-mail: blanchini@uniud.it).

R. Pesenti is with the Dip. di Matematica Applicata, Università Ca' Foscari Ca' Dolfin, Venezia E-30123, Italy.

Digital Object Identifier 10.1109/TAC.2009.2034204
Counterpart" (ARC) problem, "Two-stage Robust optimization with recourse". In many cases the adjustable variables are expressed affinely on the uncertainty and the problem is renamed "Affinely Adjustable Robust Counterpart" (AARC) problem. ARC and AARC formulations are currently a hot topic in the mathematical programming and operations research field. There are interesting connections between this paper and the notions of "adjustable variables" in ARC, AARC. For instance, the flow plays the role of the adjustable variables in the ARC set up and in most cases the strategy is affine in the uncertainty as in AARC problems.

In this paper, we address both the cases of networks without buffers and networks with buffers. If no buffers are present, incoming and outcoming flow at each site are equal since there is no stored inventory. In this case we also say that the flow balances the demand. In the case of networks with buffers, inventory accumulate at the production sites as result of the discrepancy between incoming and outcoming flows. According to existing works in the control literature [13]-[17], [21], buffers' dynamics is described by linear continuous-time differential equations.

In our model there are two types of flows, the controlled one and the uncontrolled one. For brevity, we will use the terms "flow" to mean the controlled one and "demand" to mean uncontrolled one, although there are many realistic situations in which an uncontrolled flow is not a demand. We assume that both flow and demand lie in pre-defined polytopes.

The basic problem is that of designing off-line a strategy, namely a control law for the flow. The flow will be computed on-line, on the basis of the measured buffer levels (if any) and demand by means of the provided strategy. The actual realization of the demand is not available in the design stage. In networks with buffers, we impose the buffer level to reach a prescribed level in finite time up to an assigned tolerance $\varepsilon>0$. The associated strategy is called $\varepsilon$-stabilizing (this problem is also know as "target set reachability" see [8], [9]).

We consider causal strategies of different types. Precisely we consider the case in which the flow is i) a function of the current demand (memoryless strategy), ii) a function of the current and past demand (strategy with memory), iii) a function of the buffer levels and past demand (feedback strategy), and iv) a function of the buffer levels (memoryless feedback strategy).

We deal with both a worst-case (call it also min-max) and a deterministic scenario. In the min-max (pessimistic) approach the realization maximizes the value of the given cost. In the deterministic scenario, the demand is just any arbitrary realization. Depending on the approach, the resulting strategy is said min-max or deterministically optimal respectively. 
The main novelty of this work is that we aim at optimizing a convex cost function of the long-run average-flow and demand [19]. To be more clear, in contrast with most existing literature, we are not considering the average cost of the flow (see for instance [26]) but the cost of the average flow. Clearly there is no distinction between the two concepts cost-of-the-average and average-cost in the case of linear functionals (see, e.g., [4]).

Motivations of this choice may derive from technical reasons, contracts or agreements. For instance, technical reasons or contracts may establish the long-run exploitation level of the machineries. So, over or under-exploitation of machineries can be tolerated only temporarily and not persistently. In a different situation, long-term agreements may establish privileged sources for each destination. So, a mismatch between privileged sources and destinations is acceptable only in critical and rare cases.

As a basic result we provide a constructive method to design a piecewise affine strategy which is min-max optimal. The method is based on the fact that the min-max problem arising when flow and demand are time-varying is equivalent to the min-max problem in which flow and demand are constant vectors (one-shot decisions) and not functions of time.

The obtained strategy is memoryless and such a result allows us to conclude that memory is not required when min-max optimality is considered. We initially derive these results for networks without buffers, and then extend them to networks with buffers.

In the second part of the work, we show that the provided min-max optimal strategy is not deterministically optimal, that is, it does not return the minimal cost for any given realization of the demand. Actually we show that static strategies are not deterministically optimal at all (even for networks without buffers). The second main contribution of the paper is to show that, under some smoothness assumptions on the cost functional, an easily implementable deterministically optimal strategy can be derived. Such a strategy is achieved by keeping memory of the average flow (from the initial to the current time) and by choosing, among the admissible inputs, the instantaneous minimizer of the Lyapunov derivative of the cost of the average computed up to the current time.

The structure of the paper is as follows. In Section II, we describe the problem for a network without buffers. In Section III, we determine a min-max optimal strategy. In Section IV, we extend the study to networks with buffers. In Section V, we design a deterministically optimal strategy under proper assumptions on the cost. In Section VI we provide some numerical illustrations. Finally, in Section VII we draw some conclusions.

\section{PROBlem Description}

Consider a network where at each time period the flow balances the demand. Both the flow and the demand are bounded in assigned polytopes. A simple description of such a system is

$$
\begin{aligned}
B u(t) & =w(t), \quad \forall t \\
u(t) & \in \mathcal{U}, \quad \forall t \\
w(t) & \in \mathcal{W}, \quad \forall t
\end{aligned}
$$

where $B$ is the full-row rank matrix representing the network topology, $u(t)$ is the (controlled) flow and $w(t)$ is the demand (uncontrolled flow) and $\mathcal{U} \subset \mathbb{R}^{m}$ and $\mathcal{W} \subset \mathbb{R}^{n}$ are assigned polytopes for $u$ and $w$ respectively. To let system (1)-(3) be feasible, that is to admit at least a flow $u(t)$ for any realization of $w(t)$, we must assume that (see [5] for details) the following condition holds:

$$
\mathcal{W} \subset B \mathcal{U}
$$

Given a piecewise-continuous function $f: \mathbb{R}^{+} \rightarrow \mathbb{R}^{n}$ we denote by

$$
\bar{f}_{T}=A v_{T}[f] \doteq \frac{1}{T} \int_{0}^{T} f(t) d t
$$

and

$$
\bar{f}=A v[f] \doteq \lim _{T \rightarrow \infty} A v_{T}[f]
$$

the finite-horizon and infinite-horizon average values. The simpler notation $\bar{f}$ will be often preferred where the meaning is clear from the context. In a similar way, given a sequence $f$ : $\mathcal{N} \rightarrow \mathbb{R}^{n}$, we denote by $\bar{f}=\lim _{k \rightarrow \infty}(1 / T) \sum_{k=0}^{T} f(k)$. We assume that, for any function considered in the rest of the paper, the average is defined. We generically denote by $\Phi$ a strategy of the form

$$
u=\Phi(\cdot, w(t))
$$

where the missing argument $(\cdot)$ represents any set of auxiliary variables. For instance, we admit strategies of the form

$$
\begin{aligned}
u(t) & =\Phi(\xi(t), w(t), t), \\
\dot{\xi}(t) & =\Phi_{\xi}(\xi(t), u(t), w(t), t)
\end{aligned}
$$

where $\xi$ is the controller state vector. We do not assume any special requirement for the domain of $\xi$ which can be any functional space and $\Phi_{\xi}$ can be any operator. The following assumption clarifies the information available to the network manager.

Assumption 1:

- The value $w(t)$ is available on-line at time $t$ without delay.

- The realization $w$ is not known in advance so the strategy can rely on the memory of the past values $w(\tau), \tau \leq t$, but there is no forecast about the future.

We will focus our attention to the simple case of static strategies according to the next definition.

Definition 1: The strategy $\Phi$ is called memoryless if it is a function of the current demand only, $u(t)=\Phi(\cdot, w(t)) \equiv$ $\Phi(w(t))$. Otherwise, the strategy $\Phi(\cdot, w(t))$ is called with memory.

Let $\Psi(u, w)$ be a real-valued function representing a cost. In this paper we consider different concepts of optimality as specified next. Henceforth, we use the min-max notation (rather than inf-sup) as we will prove that the problems of interests always admit a minimum and a maximum. 
Definition 2: A strategy $\Phi$ is min-max optimal (or worst-case optimal) if it is a solution of the problem

$$
\begin{array}{ll} 
& \min _{\Phi} \max _{w(\cdot)} \Psi(A v[u], A v[w]) \\
\text { s.t. } & u(t)=\Phi(\cdot, w(t)) \in \mathcal{U}, \quad \forall t, \\
& B u(t)=w(t), \quad \forall t, \\
& w(t) \in \mathcal{W}, \quad \forall t .
\end{array}
$$

Definition 3: A strategy $\Phi$ is deterministically optimal if, for any realization of $w(\cdot)$, it is a solution of the problem

$$
\begin{array}{ll} 
& \min _{\Phi} \Psi(A v[u], A v[w]) \\
\text { s.t. } & u(t)=\Phi(\cdot, w(t)) \in \mathcal{U}, \quad \forall t, \\
& B u(t)=w(t), \quad \forall t, \\
& w(t) \in \mathcal{W}, \quad \forall t .
\end{array}
$$

Remark 1: It is easy to observe that deterministically optimal strategy, if it exists, is also min-max optimal.

Given the scalar values $\alpha_{k}$, for $k=1,2, \ldots, K$ we write

$$
\text { c.c. } \alpha_{k} \text {, to mean that } \alpha_{k} \geq 0 \sum_{k=1}^{K} \alpha_{k}=1
$$

so that, given any set of vectors $w^{(k)}$, we write any convex combination as

$$
w=\sum_{k=1}^{K} \alpha_{k} w^{(k)}, \text { c.c. } \alpha_{k} .
$$

Consider the following assumption for $\Psi$.

Assumption 2: Function $\Psi$ is continuous over the set $\mathcal{U} \times \mathcal{W}$ and also convex, ${ }^{1}$ namely, for any finite set $\left\{\left(u^{(k)}, w^{(k)}\right) \mid, k=\right.$ $1, \ldots, K\}$ and c.c. $\alpha_{k}$

$$
\Psi\left(\sum \alpha_{k} u^{(k)}, \sum \alpha_{k} w^{(k)}\right) \leq \sum \alpha_{k} \Psi\left(u^{(k)}, w^{(k)}\right) .
$$

Under the above assumption we consider the following two problems.

Problem 1: Robust Problem. Determine if it exists a static min-max optimal strategy.

Problem 2: Deterministic Problem. Determine if it exists a deterministically optimal strategy.

As we will see both problems are solvable, but the strategy solution of the Deterministic Problem cannot be static.

In the current formulation, the system has no buffers, so no backlog or surplus is admitted. In Sections IV and V, we generalize the results to networks with buffers.

\section{A. A Motivating Example}

Example 1: Consider a resource distribution network whose graph is represented in Fig. 1, with unknown but bounded demand $w_{i}^{-} \leq w_{i} \leq w_{i}^{+}, i=1,2,3$ and bounded flow $0 \leq u_{i} \leq$ $u_{i}^{+}, i=1, \ldots, 12$. Constraints (1) establish a relation between demand and flow. In this example, $B$ is the $6 \times 12$ (i.e. $n=6$

\footnotetext{
${ }^{1}$ the considered concept is often referred to as "joint convexity"; note that we are not requiring just that $\Psi$ is convex in both arguments separately.
}

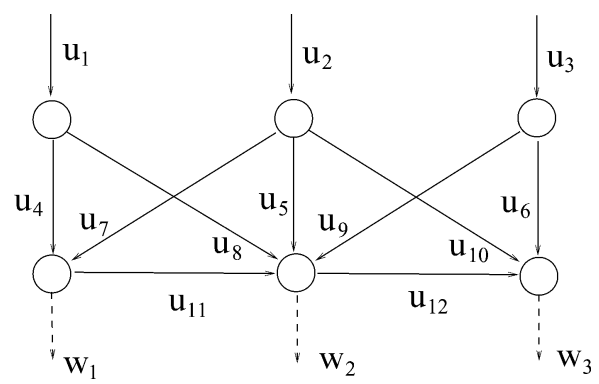

Fig. 1. Network for the example.

and $m=12$ ) incidence matrix of the network with $n=6$ nodes and $m=12$ (solid) arcs.

It is obvious that a necessary condition for the existence of a balancing flow for any admissible demand is that the maximum incoming flow $u_{\max }=u_{1}^{+}+u_{2}^{+}+u_{3}^{+}$is greater than or equal to the maximum demand $w_{\max }=w_{1}^{+}+w_{2}^{+}+w_{3}^{+}$. Assume that the network manager assigns the privileged source $u_{i}$ to each demand $w_{i}, i=1,2,3$, that is, he wishes that each demand $w_{i}$ is supplied in the long run by the corresponding flow $u_{i}$. Now, take for instance, $u_{3}^{+}=6$ and $w_{3}^{+}=7$.

If for a period $w_{3}(t)$ exceeds the value 6 , then $u_{2}$ is forced to supply an extra resource. When this occurs, we can say, roughly speaking, that $u_{2}$ is over exploited and $u_{3}$ is under exploited. So, in general, a peak of demand $w_{i}$ might require the exploitation of a flow not directly associated to that demand.

The underlying idea is to find a mechanism to balance the exploitation of $u_{2}$ and $u_{3}$ by charging $u_{3}(t)$ more than strictly necessary when $w_{3}(t)$ is low. This goal can be seen as a long-run optimization problem with cost

$$
\Psi(\bar{u}, \bar{w})=\sum_{i=1}^{3}\left(\bar{w}_{i}-\bar{u}_{i}\right)^{2}+\rho\|\bar{u}\|^{2} .
$$

The additional term is motivated by the fact that we might be also interested in avoiding high flows in the other arcs, although our theory works with $\rho=0$ as well.

\section{Min-MaX Optimality}

In this section, we consider three versions of the robust problem. The first one is formulated without restrictions on the type of strategy which can have a Memory (hence the subscript M)

$$
\begin{aligned}
\Psi_{M}= & \min _{\Phi} \max _{w(\cdot)} \Psi(A v[u], A v[w]) \\
\text { s.t. } \quad & u(t)=\Phi(\cdot, w(t)) \in \mathcal{U}, \quad \forall t, \\
& B u(t)=w(t), \quad \forall t, \\
& w(t) \in \mathcal{W}, \quad \forall t .
\end{aligned}
$$

In the second version only static strategies are admitted (i.e., Memoryless, hence the subscript ML)

$$
\begin{gathered}
\Psi_{M L}=\min _{\Phi} \max _{w(\cdot)} \Psi(A v[u], A v[w]) \\
\text { s.t. } \quad u(t)=\Phi(w(t)) \in \mathcal{U}, \quad \forall t \\
\\
B u(t)=w(t), \quad \forall t,
\end{gathered}
$$




$$
w(t) \in \mathcal{W}, \quad \forall t
$$

The third is a pure static one in which $w$ and $u$ are constant

$$
\begin{gathered}
\Psi_{S}=\min _{\Phi} \max _{w \in \mathcal{W}} \Psi(u, w) \\
\text { s.t. } \quad u=\Phi(w) \in \mathcal{U}, \\
\quad B u=w .
\end{gathered}
$$

The main result of this section is to prove that the problems of interests always admit a minimum and a maximum, and that $\Psi_{M}=\Psi_{M L}=\Psi_{S}$. The static version of the problem will enable us to determine a min-max optimal strategy. It is intuitive that the worst-case $w$ for the static strategy is assumed on the vertices. We will show that this is true also for the time-varying problem, precisely that the worst-case $w(t)$ assumes its values on the vertices.

\section{A. Solution to the Static Version of Problem 1}

Let us denote by $\operatorname{vert}\{\mathcal{W}\}$ the set of vertices of $\mathcal{W}$ and by $\mathcal{K}=\{1,2, \ldots, K\}$ the set of indices of the vertices. The static version of Problem 1 can be easily solved as follows. For each $w^{(k)} \in \operatorname{vert}\{\mathcal{W}\}$, solve the following static convex optimization problem:

$$
\begin{gathered}
u^{(k)}=\arg \min _{u} \Psi\left(u, w^{(k)}\right) \\
\text { s.t. } \quad u=\Phi\left(w^{(k)}\right) \in \mathcal{U}, \\
B u=w^{(k)} .
\end{gathered}
$$

The first lemma introduces a piecewise-affine function which will be proved to be min-max optimal. Such a function is obtained by interpolating the pairs $\left(u^{(k)}, w^{(k)}\right)$.

Lemma 1: Let $w^{(k)}$ be the vertices of $\mathcal{W}$ and $\tilde{u}^{(k)} \in \mathbb{R}^{m}$ be assigned values, for $k \in \mathcal{K}$ and let $p$ be the dimension of the set $\mathcal{W}$. There exists a partition of $\mathcal{W}$ into simplices $\mathcal{W}_{j}$ each having non-empty relative interior ${ }^{2}$ and each couple of simplices intersects at most on a $p-1$-dimensional facet. There also exists a function $\Phi(w)$ which is affine on each $\mathcal{W}_{j}$ and such that $\Phi\left(w^{(k)}\right)=\tilde{u}^{(k)}$, for each $k \in \mathcal{K}$.

For a proof see [20] and [12]. See also Example 2, where a cube is partitioned into four simplices.

The piecewise affine strategy mentioned in the previous lemma can be derived as follows. Denote by $\mathcal{K}_{j}=$ $\left\{i_{1}^{j}, i_{2}^{j}, \ldots, i_{p+1}^{j}\right\} \subseteq \mathcal{K}$ the set of the indices associated with the $j$ th simplex $\mathcal{W}_{j}$. Then for $w \in \mathcal{W}_{j}$, where $w=\sum_{i \in \mathcal{K}_{j}} \alpha_{i} w^{(i)}$, c.c. $\alpha_{i}$

$$
u=\Phi_{P W A}(w)=\sum_{i \in \mathcal{K}_{j}} \alpha_{i} u^{(i)} .
$$

For any simplex $\mathcal{W}_{j}$ the values $\alpha_{k}$ are uniquely determined, by the following set of $p+1$ equations

\footnotetext{
${ }^{2}$ a simplex in $\mathbb{R}^{n}$ is a polytope with $n+1$ vertices; its relative interior is the interior within the smallest subspace including $\mathcal{W}$; the dimension $p \leq n$ of such a subspace is the dimension of the set $\mathcal{W}$
}

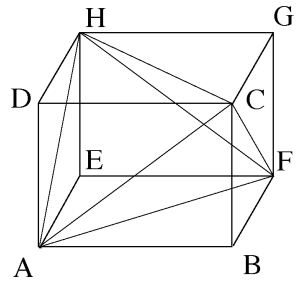

Fig. 2. Partition of a cube in four simplices.

$$
\sum_{i \in \mathcal{K}_{j}} \alpha_{i} w^{(i)}=w, \quad \sum_{i \in \mathcal{K}_{j}} \alpha_{i}=1
$$

thus the strategy is well-defined. Note that to compute the function we need to detect the sector including $w(t)$ and then to solve a linear system. Note that the matrices associated to these system can be inverted off-line to achieve an explicit expression (see [7], [12] for further details on this type of strategies).

Example 2: Assume that the demand in Example 1 is bounded in a parallelepiped, namely $w_{i}^{-} \leq w_{i} \leq w_{i}^{+}, i=1,2$, 3 . This box belongs to the subspace $w_{i}=0, i=4,5,6$, then the relative dimension is $p=3$. Such a box can be partitioned in 4 simplices as in Fig. 2 (precisely (A-C-D-H), (A-C-F-H), (A-B-C-F) and (H-C-G-F)). Denote by $\Phi_{A} \ldots \Phi_{H}$ the optimal values of $u$ in problem (12) corresponding to $w$ on the vertices $w_{A} \ldots w_{H}$. Then the interpolating affine function $u=\Phi(w)$ can be computed as follows. If, for instance, $w$ is in the sector (A-C-D-H), then

$$
\Phi(w)=\alpha_{A} \Phi_{A}+\alpha_{C} \Phi_{C}+\alpha_{D} \Phi_{D}+\alpha_{H} \Phi_{H}
$$

where $\alpha_{A}, \alpha_{C}, \alpha_{D}$ and $\alpha_{H}$ are (uniquely) determined by

$$
\begin{aligned}
\alpha_{A} w_{A}+\alpha_{C} w_{C}+\alpha_{D} w_{D}+\alpha_{H} w_{H} & =w, \\
\alpha_{A}+\alpha_{C}+\alpha_{D}+\alpha_{H} & =1 .
\end{aligned}
$$

Lemma 1 introduces the following theorem concerning the solution of the static version of Problem 1.

Theorem 1: The cost of (11) is

$$
\Psi_{S}=\max _{k \in \mathcal{K}} \Psi\left(u^{(k)}, w^{(k)}\right) .
$$

Proof: Since the $w$ in the static problem (11) can always be set as a vertex $w=w^{(k)}$, it is obvious that $\Psi_{S} \geq \max _{k \in \mathcal{K}}\left\{\Psi\left(u^{(k)}, w^{(k)}\right)\right\}$ (we remind that $u^{(k)} \in \mathcal{U}$ are the optimal values).

We show now that $\Psi_{S} \leq \max _{k \in \mathcal{K}}\left\{\Psi\left(u^{(k)}, w^{(k)}\right)\right\}$. Consider any point $w \in \mathcal{W}$ also included in the $j$ th simplex of $\mathcal{W}$, that is, $w \in \mathcal{W}_{j}$. Then, denoting by $\mathcal{K}_{j} \subset \mathcal{K}$ the subset of indices identifying the vertices of $\mathcal{W}_{j}$

$$
w=\sum_{k \in \mathcal{K}_{j}} \alpha_{k} w^{(k)}, \text { c.c. } \alpha_{k} .
$$

Take $u=\Phi_{P W A}(w)$, the piecewise-affine strategy (13). Then 


$$
\begin{aligned}
\Psi(u, w) & =\Psi\left(\sum_{k \in \mathcal{K}_{j}} \alpha_{k} u^{(k)}, \sum_{k \in \mathcal{K}_{j}} \alpha_{k} w^{(k)}\right) \\
& \leq \sum_{k \in \mathcal{K}_{j}} \alpha_{k} \Psi\left(u^{(k)}, w^{(k)}\right) \leq \max _{k \in \mathcal{K}_{j}}\left\{\Psi\left(u^{(k)}, w^{(k)}\right)\right\} \\
& \leq \max _{k \in \mathcal{K}}\left\{\Psi\left(u^{(k)}, w^{(k)}\right)\right\}
\end{aligned}
$$

with c.c. $\alpha_{k}(t)$. Note that the last inequality compares the maximum over all vertices of $\mathcal{W}_{j}$ with the maximum over all vertices of $\mathcal{W}$.

An immediate consequence of the above theorem is that the strategy $\Phi_{P W A}(w)$ in (13) is min-max optimal for the static problem (11) as it guarantees that

$$
\begin{aligned}
\Psi\left(\Phi_{P W A}(w), w\right) & \leq \Psi\left(\Phi_{P W A}\left(w^{(r)}\right), w^{(r)}\right) \\
& =\Psi\left(u^{(r)}, w^{(r)}\right)
\end{aligned}
$$

where $w^{(r)}$ is the worst demand. Note that this strategy is not unique since all the strategies in the set

$Q=\{\Phi(w): B \Phi(w)=w$,

$\left.\Psi(\Phi(w), w) \leq \Psi\left(\Phi_{P W A}\left(w^{(r)}\right), w^{(r)}\right), \forall w \in \mathcal{W}\right\}$

are min-max optimal for the static problem (11). In particular, the set $Q$ includes the strategy

$$
\Phi_{S O P T}(w)=\arg \min _{u \in \mathcal{U}}\{\Psi(u, w): B u=w, \forall w \in \mathcal{W}\} .
$$

Remark 2: Note that the $u$ selected by (16) is deterministically optimal for the pure static problem only, in the sense that $\Psi\left(\Phi_{S O P T}(w), w\right) \leq \Psi(u, w)$ for all $w \in \mathcal{W}$ and $u \in \mathcal{U}$. However, the same $u$ is, in general, not optimal for the Deterministic Problem (Problem 2). Both $\Phi_{S O P T}(w)$ and $\Phi_{P W A}(w)$ are max-min optimal (in the game-theoretic language the "demand plays first" [3]), in the sense that the flow is taken as a function of $w$ which, in turn, maximizes the cost $\Psi\left(\Phi_{S O P T}(w), w\right)$ in one of the vertices $w^{(r)}$. So an interpretation of our result is that to design a min-max optimal strategy off-line we can solve a max-min problem on-line, i.e., find minimizing flow for given demand.

\section{B. Main Theorem}

We are in a position to prove that the optimal min-max cost is equal in the three versions (9)-(11) of the Robust Problem. Basically, we prove that the worst-case demand for the static problem, namely the value $w^{(r)} \in \mathcal{W}$ on which the maximum (14) is assumed, is indeed the worst-case demand for the timevarying problem that we are considering.

Theorem 2: The following equalities hold:

$$
\Psi_{M}=\Psi_{M L}=\Psi_{S} .
$$

Furthermore, as the inf-sup optimal values are achieved with strategy $\Phi_{P W A}(w)$ in all the three problems (9), (10) and (11), then $\Psi_{M}, \Psi_{M L}$ and $\Psi_{S}$ are min-max optimal values and the static strategy $\Phi_{P W A}(w)$ is a min-max optimal strategy.
Proof: The proof splits in two parts.

Proof of the claim $\Psi_{M L}=\Psi_{S}$. Let $w^{(r)} \in \operatorname{vert}\{\mathcal{W}\}$ the worst-case demand for the static problem (11), and let $u^{(r)}$ the corresponding (optimal) flow. Let $u=\Phi(w)$ any arbitrary memoryless strategy. Assume that the demand is constant $w(t) \equiv w^{(r)}$ and let $\hat{u}=\Phi\left(w^{(r)}\right)$. Since $u$ and $w$ are constant, the average cost is

$$
\Psi\left(\bar{u}, w^{(r)}\right)=\Psi\left(\hat{u}, \bar{w}^{(r)}\right) \geq \Psi\left(u^{(r)}, \bar{w}^{(r)}\right)=\Psi_{S}
$$

by construction, so we have $\Psi_{M L} \geq \Psi_{S}$.

We now prove $\Psi_{M L} \leq \Psi_{S}$. For arbitrary $w(t)$ and $u(t)=$ $\Phi_{P W A}(w(t))$, consider the averages $\bar{u}_{T}=A v_{T}[u]$ and $\bar{u}_{T}=$ $A v_{T}[w]$ the associated cost is

$$
\begin{aligned}
\Psi & \left(\bar{u}_{T}, \bar{w}_{T}\right) \\
& =\Psi\left(\frac{1}{T} \int_{0}^{T} \sum_{k \in \mathcal{K}} \alpha_{k}(t) u^{(k)} d t, \frac{1}{T} \int_{0}^{T} \sum_{k \in \mathcal{K}} \alpha_{k}(t) w^{(k)} d t\right) \\
& =\Psi\left(\sum_{k \in \mathcal{K}} u^{(k)} \frac{1}{T} \int_{0}^{T} \alpha_{k}(t) d t, \sum_{k \in \mathcal{K}} w^{(k)} \frac{1}{T} \int_{0}^{T} \alpha_{k}(t) d t\right) \\
& =\Psi\left(\sum_{k \in \mathcal{K}} u^{(k)} \hat{\alpha}_{k}(T), \sum_{k \in \mathcal{K}} w^{(k)} \hat{\alpha}_{k}(T)\right)
\end{aligned}
$$

with c.c. $\alpha_{k}(t)$. Note that in (18) the sums are extended to all vertices of $\mathcal{W}$ but, at each time period, only the non-zero $\alpha_{k}(t)$ are those associated with current "active simplex" $\mathcal{W}_{j}$, i.e. the one for which $w(t) \in \mathcal{W}_{j}$. It is obvious that the numbers

$$
\hat{\alpha}_{k}(T)=\frac{1}{T} \int_{0}^{T} \alpha_{k}(t) d t
$$

are c.c.. Consider inside $\mathcal{U} \times \mathcal{W}$ the polytope having vertices $\left(u^{(k)}, w^{(k)}\right)$. Since $\Psi$ is convex, it reaches the maximum on its vertices then for all $T$

$$
\Psi\left(\bar{u}_{T}, \bar{w}_{T}\right) \leq \max _{k \in \mathcal{K}} \Psi\left(u^{(k)}, w^{(k)}\right)=\Psi_{S} .
$$

If we take the limit over an infinite horizon by continuity we have

$$
\Psi(A v[u], A v[w]) \leq \Psi_{S}
$$

or, which is the same, $\Psi_{M L} \leq \Psi_{S}$ and therefore we can conclude that $\Psi_{M L}=\Psi_{S}$.

Proof of the claim $\Psi_{M}=\Psi_{M L}$. Memoryless strategies are special cases of the strategies with memory, and cannot do any better, thus $\Psi_{M} \leq \Psi_{M L}$. We only have to show that $\Psi_{M} \geq$ $\Psi_{M L}$. Again we assume that $w(t) \equiv w^{(r)}$, the worst-case demand. The optimal strategy is, again, to take $u=u^{(r)}$. Indeed for each $T$ and any $u(t) \in \mathcal{U}$

$$
\begin{aligned}
\Psi\left(\frac{1}{T} \int_{0}^{T} u(t) d t, \frac{1}{T} \int_{0}^{T} w^{(r)} d t\right) & =\Psi\left(A v_{T}[u], w^{(r)}\right) \\
& \geq \Psi\left(u^{(r)}, w^{(r)}\right)
\end{aligned}
$$


where the last inequality comes from the fact that the average $\bar{u}_{T} \in \mathcal{U}$ and $u^{(r)}$ is optimal by construction. Then $\Psi_{M} \geq \Psi_{S}=$ $\Psi_{M L}$ and therefore $\Psi_{M}=\Psi_{M L}$.

Remark 3: The minimizer in the Robust Problems (9)-(11) chooses strategies and not flows (the min is over $\Phi(\cdot)$ and not $u(\cdot))$ and this justifies the fact that, as the worst demand is on a vertex, then the optimal flow $\Phi$ turns out to be a function which interpolates the optimal values $u^{(r)}=\Phi\left(w^{r}\right)$ associated with the vertices $w^{(r)}$. Clearly $u^{(r)}$ need not to be a vertex of $\mathcal{U}$.

So far, we have assumed that the average values of the realization of $w$ and the flow computed by the strategy $\Phi$ always exist. The following remark points out that we may drop such an assumption.

Remark 4: In view of (20), it is always possible to prove that

$$
\lim \sup _{T \rightarrow \infty} \Psi\left(A v_{T}[u], A v_{T}[w]\right) \leq \Psi_{S} .
$$

Therefore if we rewrite the Robust Problem in the "lim-sup version" we achieve that

$$
\begin{array}{ll} 
& \Psi_{L S} \doteq \min _{\Phi} \max _{w \in \mathcal{W}} \lim \sup _{T \rightarrow \infty} \Psi\left(A v_{T}[u], A v_{T}[w]\right), \\
\text { s.t. } & \Phi(\cdot, w(t)) \in \mathcal{U}, \quad B u=w
\end{array}
$$

is equal to $\Psi_{S}$ no matter which type of strategy is chosen.

The following remark generalizes the results of this section to the discrete-time case. It will turn useful later on when we study networks with buffers.

Remark 5: Theorem 2 still holds if we state problems (9) and (10) in discrete-time with $t=0,1,2, \ldots$.

\section{The CASE of Networks With Buffers}

The first part of this work is based on the assumption that at each time period the flow balances the demand. We now drop such an assumption and assume that the unbalanced flow is stored at the node buffers. We wish to find a min-max optimal strategy that steers the buffer levels to an arbitrarily small set in finite time, and keeps them bounded in the small set from that time on. Then, the new formulation (network with buffers) is identical to that of Problem 1 if we replace (9) by

$$
\begin{aligned}
\Psi_{B}= & \min _{\Phi} \max _{w(\cdot)} \Psi(A v[u], A v[w]) \\
\text { s.t. } & u(t)=\Phi(\cdot, x(t), w(t)) \in \mathcal{U}, \quad \forall t, \\
& w(t) \in \mathcal{W}, \quad \forall t, \\
& \dot{x}(t)=B u(t)-w(t), \quad \forall t, \\
& x(t) \in \mathcal{X}, \quad \forall t, \\
& x(t) \in \varepsilon \mathcal{X}, \quad \forall t \geq t_{f} \text { for some } t_{f}>0
\end{aligned}
$$

where the vector $x(t)$ describes the buffer levels, the bounding set $\mathcal{X}$ is convex and compact and includes the origin as an interior point, and the arbitrarily small set $\varepsilon \mathcal{X}$, with $\varepsilon>0$, is the set within which $x$ must be driven in finite time. Henceforth, given a generic set $\mathcal{A} \subseteq \mathbb{R}^{n}$ and being $\lambda$ a positive scalar, we denote by $\lambda \mathcal{A}=\{\lambda a: a \in \mathcal{A}\}$. The strategies $\Phi$ considered are of the form

$$
u(t)=\Phi(\xi(t), x(t), w(t), t),
$$

$$
\dot{\xi}(t)=\Phi_{\xi}(\xi(t), x(t), u(t), w(t), t)
$$

again, with no restrictions of the type of domain of the variables. Before presenting the solution of problem (21), we need to discuss certain feasibility conditions for it and some technical assumptions for a "nice" description of the bounding sets $\mathcal{X}$.

Problem (21) is feasible if and only if the following condition holds [14]

$$
\mathcal{W} \subset \operatorname{int}\{B \mathcal{U}\}
$$

where $\operatorname{int}\{B \mathcal{U}\}$ means the interior part of set $B \mathcal{U}$. The above condition is stronger than condition $\mathcal{W} \subset B \mathcal{U}$ considered in the first part of this work. We also assume, without restriction, that

$$
0 \in \mathcal{W} \text { and } 0 \in \operatorname{int}\{\mathcal{U}\}
$$

(we can always apply a proper translation to meet this assumption). As an immediate consequence of (23) and (24), we can affirm that there exists a scalar $0<\sigma<1$, such that

$$
\mathcal{W} \subset(1-\sigma)\{B \mathcal{U}\} .
$$

Regards to the description of the bounding set $\mathcal{X}$, we make the following assumption.

Assumption 3: There exists a gauge function $\psi$ which is smooth for $x \neq 0$ and such that

$$
\mathcal{X}=\{x: \psi(x) \leq 1\} .
$$

Let us remind that gauge functions are positive definite, convex, and positively homogeneous of order 1, i.e., such that $\psi(\xi x)=$ $\xi \psi(x)$ for $\xi \geq 0$ [22]. Special gauge functions $\psi$ are $\|x\|$ or more in general $\|F x\|_{2 p}$ with $F$ full column rank and integer $p \geq 1$. Note that norms of the type $\|F x\|_{\infty}$ may be arbitrarily closely approximated by $\|F x\|_{2 p}$ for $p$ large. Then, non-smooth polytopic sets of the form $\mathcal{X}=\{x: F x \leq \overline{1}\}$, where $\overline{1}=$ $\left[\begin{array}{lll}1 & 1 \ldots 1\end{array}\right]^{T}$ defined by functions of the type

$$
\psi(x)=\max _{k} \quad F_{k} x
$$

where $F_{k}$ is the $k$ th row of $F$, can be approximated by smooth sets $\hat{\mathcal{X}}$ defined by functions of the type

$$
\hat{\psi}(x)=\left[\sum_{k}\left(\max \left\{F_{k} x, 0\right\}\right)^{p}\right]^{1 / p} .
$$

We are now in a position to establish the main result of this section which says that problem (21) is equivalent to problem (10) and therefore to problems (9) and (11). It is apparent that, given the presence of buffers and no constraints on the initial value $x(0)$ of their levels, a strategy memoryless or with memory as in Definition 1 cannot solve problem (21) since buffer level feedback is necessary. To this end, let us introduce the following definitions.

Definition 4: The strategy is of the pure feedback form if $u(t)=\Phi(\cdot, x(t))$, namely it requires no information about the current value of $w(t)$. The strategy is of the memoryless pure feedback form if $u(t)=\Phi(x(t))$, namely it is function only of the current value of the buffer levels $x(t)$. 
The following theorem states that there exist strategies with a simple structure that solve problem (21).

Theorem 3: The following property holds:

$$
\Psi_{B}=\Psi_{M L}
$$

Furthermore, min-max optimal strategies can be obtained either adding a memoryless pure feedback component to a static strategy, that is $\Phi(x(t), w(t))=\Phi_{1}(w(t))+\Phi_{2}(x(t))$, or through a pure feedback strategy $\Phi(\cdot, x(t))$.

Proof: We first prove (25) under the assumption that $w(t)$ is known on-line. To do this, consider the following strategy:

$u(t)=\Phi(x(t), w(t))=\Phi_{1}(w(t))+\Phi_{2}(x(t))$, for all $t \geq 0$

where $\Phi_{1}(w(t))$ is any static strategy, for instance $\Phi_{P W A}(w(t))$, which solves problem (10), and $\Phi_{2}(x)$ is a stabilizing term that we define later on. Note that

$$
B \Phi_{1}(w(t))=w(t) \in(1-\sigma) B \mathcal{U}
$$

then if we substitute $u(t)$ in the state equation in problem (22) we get

$$
\dot{x}(t)=B \Phi(x, w)-w(t)=B \Phi_{2}(x) .
$$

Now take $\Phi_{2}(x)$ equal to the continuous function

$$
\Phi_{2}(x)=\operatorname{sat}_{\sigma}\left[-B^{\dagger} x\right]
$$

where $B^{\dagger}$ is any right inverse of $B$ and the saturation function is defined as follows:

$$
\operatorname{sat}_{\sigma}\left[-B^{\dagger} x\right]=-\lambda(x) B^{\dagger} x
$$

with

$$
\lambda(x) \doteq \max \left\{\tilde{\lambda} \geq 0: \quad-\tilde{\lambda} B^{\dagger} x \in \sigma \mathcal{U}\right\}
$$

Note that this assures that $\Phi(x, w)=\Phi_{1}(w)+\Phi_{2}(x) \in \mathcal{U}$. Since $\sigma \mathcal{U}$ includes 0 as an interior point we have that the Lyapunov derivative of $\psi$ is negative for $x \neq 0$. Indeed, since for gauge functions $\nabla \psi(x) x=\psi(x)$ holds for $x \neq 0$, we have

$$
\begin{aligned}
\dot{\psi}(x) & =\nabla \psi(x) B \Phi_{2}(x)=-\nabla \psi(x) B B^{\dagger} \lambda(x) x \\
& =-\lambda(x) \psi(x)<0
\end{aligned}
$$

for $x \neq 0$. Therefore

$$
x(t) \rightarrow 0
$$

(see [14] for details). Note that this means that, by continuity, $\Phi_{2}(x(t))$ converges to zero and thus it has zero average. This also means that

$$
A v[u(t)]=A v\left[\Phi_{1}(w(t))\right]
$$

It remains to prove that this strategy solves problem (21). Consider the average

$$
\begin{aligned}
A v[u] & =\lim _{T \rightarrow \infty} \frac{1}{T} \int_{0}^{T} u(t) d t \\
& =\lim _{T \rightarrow \infty} \frac{1}{T} \int_{0}^{T} \Phi_{1}(w(t)) d t+\lim _{T \rightarrow \infty} \frac{1}{T} \int_{0}^{T} \Phi_{2}(x(t)) d t \\
& =\lim _{T \rightarrow \infty} \frac{1}{T} \int_{0}^{T} \Phi_{1}(w(t))=A v\left[\Phi_{1}(w(t))\right] .
\end{aligned}
$$

Then by repeating exactly the same arguments of the previous section we have that the first part of the theorem is proved.

We now show that a pure feedback robust strategy $u(t)=$ $\Phi(\cdot, x(t))$ exists, i.e., a robust strategy that does not require the knowledge of the current value of $w(t)$. This can be done by sampling the system at small intervals. Actually, let $\tau>0$ and consider the following equation:

$$
x(k)=x(k-1)+B \int_{(k-1) \tau}^{k \tau} u(t) d t-\int_{(k-1) \tau}^{k \tau} w(t) d t .
$$

Let us now introduce the discrete-time variable $z$ defined as

$$
z(k)=x(k-1)+B \int_{(k-1) \tau}^{k \tau} u(t) d t
$$

equation (26) yields

$$
z(k)-x(k)=\int_{(k-1) \tau}^{k \tau} w(t) d t \doteq \tau \hat{w}(k)
$$

where $\hat{w}(k) \in \mathcal{W}$ is the average demand in $[(k-1) \tau, k \tau]$. This means that we can derive the integral of $w$ over $[(k-1) \tau, k \tau]$ by simply computing $z(k)$ and measuring $x(k)$.

The idea is now to apply a piecewise constant flow which takes on the following constant value within each sampling interval $[k \tau,(k+1) \tau)$

$$
u(k)=\hat{\Phi}(z(k), x(k))=\hat{\Phi}_{1}(z(k), x(k))+\hat{\Phi}_{2}(z(k))
$$

$k \tau \leq t<(k+1) \tau$, where $\hat{\Phi}_{1}$ is meant to compensate the past demand and $\hat{\Phi}_{2} \in \sigma \mathcal{U}$ is a feedback action. In particular, the term $\hat{\Phi}_{1}$ is chosen in such a way that

$$
\begin{aligned}
\tau B \hat{\Phi}_{1}(z(k), x(k)) & =\tau \hat{w}(k)=z(k)-x(k), \\
\hat{\Phi}_{1}(z(k), x(k)) & \in(1-\sigma) \mathcal{U}
\end{aligned}
$$

The above condition is satisfied by

$$
\hat{\Phi}_{1}(z(k), x(k))=\Phi(\hat{w}(k))
$$

where $\Phi(w)$ is any memoryless strategy which solves problem (10) in its discrete-time version as defined in Remark 5. We just have to assume that the demand values $w(t)$ for $t=0,1,2, \ldots$ 
are equal to $\hat{w}(k)$ for $k \tau=t$. To specify the term $\hat{\Phi}_{2} \in \sigma \mathcal{U}$, first note that the latter equation together with (27) yields

$$
\begin{aligned}
z((k+1)) & =x(k)+\tau B \hat{\Phi}(z(k), x(k)) \\
& =x(k)+\tau B \hat{\Phi}_{1}(z(k), x(k))+\tau B \hat{\Phi}_{2}(z(k)) \\
& =z(k)+\tau B \hat{\Phi}_{2}(z(k))
\end{aligned}
$$

then, select $\hat{\Phi}_{2}(z(k))$ according to

$$
\hat{\Phi}_{2}(z(k))= \begin{cases}\arg \min _{u \in \sigma \mathcal{U}} \psi(z(k)+\tau B u) & \text { if } z \neq 0 \\ 0 & \text { if } z=0 .\end{cases}
$$

Note that if we use $\psi(z(k))$ as discrete-time Lyapunov function, the condition $0 \in \operatorname{int}\{\mathcal{U}\}$ guarantees that $\psi(z(k+1))-$ $\psi(z(k)) \leq-\beta<0$ until the rest condition $\psi(z(\vec{k}))=0$ is reached for some large enough but finite $\tilde{k}$. As a consequence $x(k)=z(k)-\tau \hat{w}(k)$ is ultimately bounded in the set $\tau \mathcal{W}$. By assuming $\tau$ small enough we can drive $x(k)$ inside $\varepsilon \mathcal{X}$. Given this, since $z(k)=0$ for any $k \geq \tilde{k}$, the feedback strategy $\hat{\Phi}_{2}(z(k))$ is equal to zero then $A v\left[\hat{\Phi}_{2}(z(k))\right]=0$. Now, consider the average value of $u(t)$

$$
\begin{aligned}
A v[u]= & \lim _{T \rightarrow \infty} \frac{1}{T} \int_{0}^{T} u(t) d t=\lim _{K \rightarrow \infty} \frac{1}{K \tau} \sum_{k=0}^{K-1} \int_{k \tau}^{(k+1) \tau} u(t) d t \\
= & \lim _{K \rightarrow \infty} \frac{1}{K} \sum_{k=0}^{K-1} \hat{\Phi}_{1}(z(k), x(k)) \\
& +\lim _{K \rightarrow \infty} \frac{1}{K} \sum_{k=0}^{K-1} \hat{\Phi}_{2}(z(k)) \\
= & A v\left[\hat{\Phi}_{1}(z(k), x(k))\right] .
\end{aligned}
$$

We have just proved that we can choose a strategy such that $A v[u]=A v\left[\hat{\Phi}_{1}\right]=A v[\Phi]$ where $\Phi$ is a memoryless strategy solution of problem (10). Then, according to Remark 5, we have that $\Psi_{B} \leq \Psi_{M L}$. It is left to prove that $\Psi_{B} \geq \Psi_{M L}$, or, which is the same, $\Psi_{B} \geq \Psi_{S}$. To do this, let us denote by $w^{(r)}$ the worst demand of the static problem (11). Assuming that $w(t) \equiv w^{(r)}$, an optimal strategy for $u$ is to take $u(t) \equiv \Phi_{1}\left(w^{(r)}\right)$ as already shown by (20) in the proof of Theorem 2 .

Remark 6: In the developed theory we assumed that the time required for state measurements and flow computation is negligible. If these operations introduce a delay $\tau_{d}$, we can provide a sample-data reformulation of the problem and our results still hold. In particular, we can guarantee practical stability within some $\varepsilon$-ball with $\varepsilon$ depending on $\tau_{d}$.

\section{Deterministic Versus Min-Max Optimality}

In this section, we tackle the Deterministic Problem where the demand is a generic one and not the worst one. In particular, we remind that we wish to find a strategy $\Phi(\cdot, w(t))$ that for any realization of the demand solves the problem

$$
\begin{aligned}
\Phi_{D}= & \min _{\Phi} \Psi(A v[u], A v[w]) \\
\text { s.t. } \quad u(t)=\Phi(\cdot, w(t)) \in \mathcal{U}, \quad \forall t, & \\
& B u(t)=w(t), \quad \forall t, \\
& w(t) \in \mathcal{W}, \quad \forall t .
\end{aligned}
$$

The index D stands for "deterministic". The main result of this section is to prove that $\Phi_{P W A}(w(t))$, and in general $\Phi_{S O P T}(w(t))$ are not deterministically optimal. In particular, we find a strategy with memory that performs strictly better than the strategy $\Phi_{S O P T}(w(t)) \in Q$ as in (16) for a simple counterexample where $\Psi(A v[u])$ is a function of $A v[u]$ only and is positively homogeneous. Conversely we will present a theorem which shows that to achieve the deterministic optimality we must resort to strategies with memory, i.e., given by differential equations, notwithstanding the fact that our model is described by algebraic equations. We prove our results under the following assumption.

Assumption 4: Function $\Psi$ depends on $A v[u]$ only and it is convex and positive semidefinite. Furthermore $\Psi$ is continuously differentiable in all points $u$ for which $\Psi(u)>0$.

Before providing the counterexample and the theorem, we would like to provide the following comments about the previous assumption.

- Given any convex function which is positive semidefinite, we can always approximate it by a smooth one over an arbitrarily large compact set as we have seen in Section IV.

- The requirement that $\Psi$ is a function only of $u$ is not a restriction. Indeed, we can make $\Psi$ implicitly depend on $w$ by reviewing $w$ as additional components of $u$ as expressed below

$$
\begin{aligned}
B u-\tilde{u} & =0, \\
\tilde{u} & =w .
\end{aligned}
$$

- We can generalize the cost in a significant way by translating $u$ and considering costs that are positive semidefinite with respect to a nominal flow $u_{0}$ satisfying the nominal demand $w_{0}$, namely of the form $\Psi\left(u-u_{0}\right)$.

We consider a strategy with memory $u(t)=\Phi(\xi(t), w(t))$ as in (22) where $\xi(t)$ and the strategy $\Phi$ are defined as

$$
\begin{aligned}
\xi(t) & =\left\{\begin{array}{ll}
u(0) & t=0 \\
\frac{1}{t} \int_{0}^{t} u(\tau) d \tau & t>0
\end{array} \Leftrightarrow \dot{\xi}(t)=-\frac{\xi(t)}{t}+\frac{u(t)}{t},\right. \\
u_{G}(t) & =\Phi(\xi(t), w(t))=\arg \min _{v \in \mathcal{U}: B v=w(t)} \nabla \Psi(\xi(t)) v .
\end{aligned}
$$

As a first observation, note that the time derivative of $\Psi(\xi(t))$, for the current value of $\xi(t)$, turns out to be

$$
\dot{\Psi}(\xi(t))=\nabla \Psi(\xi(t)) \dot{\xi}(t)=\nabla \Psi(\xi(t))\left(-\frac{\xi(t)}{t}+\frac{u(t)}{t}\right) .
$$

Therefore, $u_{G}$ is the point-wise minimizer of such a derivative. The rationale of this choice is that it trivially holds $A v_{T}[u]=$ $\xi(T)$ and therefore, at any time $t$, an approximation $\Psi(\xi(t))$ of the cost $\Psi(A v[u])$ is available. The approximation is based on the average up to time $t$ rather than on the long run average. Now, we select a strategy that, at time $t$, chooses among all possible flows that balance the current demand $w(t)$, the one that induces a maximum decrease for the approximated cost. We will refer to this strategy as the Gradient-based strategy (hence the $\mathrm{G}$ in $\Phi_{G}$ ).

In the case of a non-differentiable $\Psi$ we would have to replace the Lyapunov derivative by the generalized derivative

$$
D^{+} \Psi(\xi(t))=\lim _{d t \rightarrow 0^{+}} \sup \frac{\Psi(\xi(t))-\Psi(\xi(t-d t))}{d t}
$$




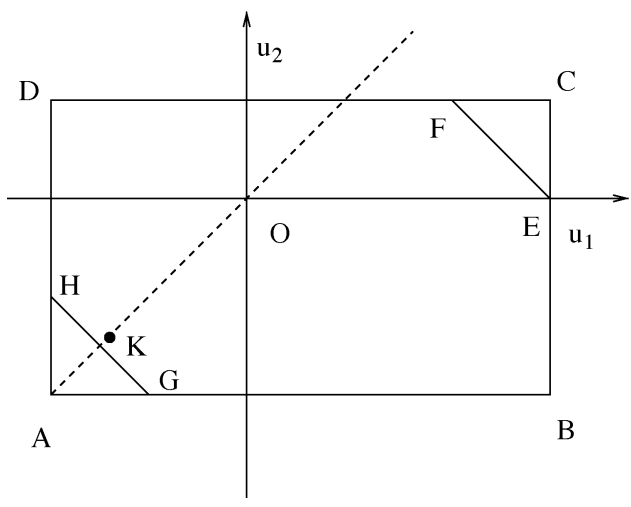

Fig. 3. Flow space of $u_{1}-u_{2}$ for the system of Example 3. The rectangle $A-B-C-D$ defines the set $\mathcal{U}$.

at the price of a much harder exposition (see for instance [12]).

We provide a simple example showing that the strategy (29) works better than the $\Phi_{S O P T}(w(t))$ which means that static strategies may be min-max optimal but they are not deterministically optimal in general.

Example 3: Let us consider the simple example proposed in [5], Example 7, with one node and two arcs. The system is described by

$$
u_{1}(t)+u_{2}(t)=w(t)
$$

with flow and demand subject to constraints $-2 \leq u_{1} \leq 3$, $-2 \leq u_{2} \leq 1$ and $|w| \leq 2.8$.

Fig. 3 displays the flow space of $u_{1}$ and $u_{2}$. The rectangle $A-B-C-D$ defines the set $\mathcal{U}$. Let the cost function be $\Psi=\left|A v\left[u_{1}\right]-A v\left[u_{2}\right]\right|$, as we wish to have the same degree of long-term exploitation of $u_{1}$ and $u_{2}$. All points for which $\Psi(\cdot)$ is zero are described by the dashed line intersecting points $A$ and $O$.

Consider a realization such that $w(t)=2.8$ for $(2 k) \Theta \leq t<$ $(2 k+1) \Theta$ and $w(t)=-2.8$ for $(2 k+1) \Theta \leq t<(2 k+2) \Theta$, for some dwell time $\Theta>0, k=0,1, \ldots$. In particular, as the realization is periodic, we can limit ourselves to study the evolution of the approximate cost in the first period (for $k=0$ ), the latter including the intervals $0 \leq t<\Theta$ where $w(t)=2.8$ and $\Theta \leq t<2 \Theta$ where $w(t)=-2.8$. We will show that in the first interval, we are not able to exploit the $u_{1}$ and $u_{2}$ at the same degree, as any feasible solution is such that $u_{2}$ works harder than $u_{1}$. In the second interval, the strategy (29) recovers the mismatch by initially letting $u_{2}$ working harder than $u_{1}$.

To be more precise, in the first interval, where $w(t)=2.8$, we must have $u_{1}+u_{2}=2.8$ (i.e., the segment $E-F$ in Fig. 3) and strategy (29) returns $u_{1}=1$ and $u_{2}=1.8$ (i.e., the point $F$, which is the closest one to the dashed line $u_{1}=u_{2}$ ). During the interval function $\Psi(\xi(t))=0.8 t$ and at the end of the first interval, for $t=\Theta$, we have $\Psi(\xi(t))=0.8 \Theta$.

In the second interval, where $w(t)=-2.8$, we must have $u_{1}+u_{2}=-2.8$ (i.e., the segment $G-H$ ) and the strategy (29) returns $u_{1}=-2$ and $u_{2}=-0.8$ (i.e., the point $H$ ) in order to drive the approximate cost to zero as fastest as possible. Let $\bar{t}$ be the first instant where $\Psi(\xi(t))=0$. We have that for $\Theta \leq t<\bar{t}$, the function $\Psi(\xi(t))=(0.8 \Theta-1.2(t-\Theta)) / t$. If we impose $\Psi(\xi(t))=0$ in the latter equation we find $\bar{t}=(5 / 3) \Theta$. In the remaining interval, namely for $\bar{t} \leq t<2 \Theta$, the function takes on the value $\Psi(\xi(t))=0$ and the strategy (29) switches to the flow $u_{1}=u_{2}=-(7 / 5)$ (point $K$ ).

As in $t=2 \Theta, \Psi(\xi(t))=0$ the above reasoning can be applied for $k=1$ and so on. We have proved that $\Psi(\xi(t))$ is bounded, $0 \leq \Psi(\xi(t)) \leq(0.8 / t) \Theta$ for all $t \geq 0$. This means that $\Psi(\xi(t)) \rightarrow 0$ for $t \rightarrow \infty$. Since, $\Psi(\xi(t)) \rightarrow \Psi(A v[u])$ also holds, we have shown that $\Psi(A v[u])=0$ and then the strategy (29) is deterministically optimal for this example. Conversely, the cost associated to $\Phi_{S O P T}(w(t))$ is strictly greater than zero which means that $\Phi_{S O P T}(w(t))$ is not deterministically optimal. Actually, when $w(t)=2.8$, the strategy $\Phi_{S O P T}(w(t))$ returns $u_{1}=1$ and $u_{2}=1.8$ (i.e., the point $F$ ), whereas when $w(t)=-2.8$, the strategy $\Phi_{S O P T}(w(t))$ returns $u_{1}=-1.4$ and $u_{2}=-1.4$ (i.e., the point $K$ ). On the average we have

$$
A v\left[u_{1}\right]=-0.2, \quad A v\left[u_{2}\right]=0.2, \quad \Phi\left(A v\left[u_{1}\right], A v\left[u_{2}\right]\right)=0.4 .
$$

The deterministic optimality of (29) is proven next.

Theorem 4: Assume that $\Psi$ satisfies Assumption 4. Assume that the gradient-based strategy (29) produces the average $\bar{u}_{G}=$ $A v\left[u_{G}\right]$. Let $\hat{u}$ the average achieved by some arbitrary strategy such that $B u(t)=w(t)$ for all $t$. Then

$$
\Psi\left(\bar{u}_{G}\right) \leq \Psi(\hat{u}) .
$$

Proof: To prove that $\Psi\left(\bar{u}_{G}\right) \leq \Psi(\hat{u})$, we first note that if $\Psi\left(\bar{u}_{G}\right)=0$ the result is straightforward since $\Psi$ is positive semidefinite. Therefore assume $\Psi\left(\bar{u}_{G}\right)>0$. Denote by $\xi_{G}(t)$ the evolution of the integral variable with the gradient-based strategy. Since $u_{G}$ is the minimizer we must have

$$
\nabla \Psi\left(\xi_{G}(t)\right) u(t) \geq \nabla \Psi\left(\xi_{G}(t)\right) u_{G}(t)
$$

for any other $u(t) \in \mathcal{U}$ such that $B u(t)=w(t)$.

Assume, by contradiction, that there exists a control $u(t) \in \mathcal{U}$ such that $B u(t)=w(t)$ and that $\Psi(\hat{u})<\Psi\left(\bar{u}_{G}\right)$. Since $\Psi$ is convex, there exists $\beta^{\prime}>0$ such that

$$
\nabla \Psi\left(\bar{u}_{G}\right)\left[\hat{u}-\bar{u}_{G}\right]<-\beta^{\prime} .
$$

Since $\bar{u}=A v\left[u_{G}\right]$ and $\hat{u}=A v[u]$ are the average values, so that $A v\left[u-u_{G}\right]=\hat{u}-\bar{u}$ it turns out that for any $\tau>0$, no matter how large, and any $0<\beta<\beta^{\prime}$ there exists $t \geq \tau$ such that

$$
\nabla \Psi\left(\bar{u}_{G}\right)\left[u(t)-u_{G}(t)\right]<-\beta .
$$

Consider now the expression

$$
\begin{aligned}
\nabla \Psi & \left(\xi_{G}(t)\right) u(t) \\
= & \nabla \Psi\left(\xi_{G}(t)\right) u_{G}(t)+\nabla \Psi\left(\xi_{G}(t)\right)\left(u(t)-u_{G}(t)\right) \\
= & \nabla \Psi\left(\xi_{G}(t)\right) u_{G}(t)+\underbrace{\nabla \Psi\left(\bar{u}_{G}\right)\left(u(t)-u_{G}(t)\right)}_{<-\beta \text { for } t \geq \tau} \\
& +\underbrace{\left[\nabla \Psi\left(\xi_{G}(t)\right)-\nabla \Psi\left(\bar{u}_{G}\right)\right]\left(u(t)-u_{G}(t)\right)}_{\rightarrow 0}
\end{aligned}
$$

where the last term goes to 0 in view of the continuity of $\nabla \Psi$ and the boundedness of $u$ and $u_{G}$. Then for a proper $t$ large enough

$$
\nabla \Psi\left(\xi_{G}(t)\right) u(t)<\nabla \Psi\left(\xi_{G}(t)\right) u_{G}(t)
$$

in contradiction with (30). 
TABLE I

LOWER BOUNDS $L B$ AND UPPER BOUNDS $U B$ OF DEMAND AND FLOW

\begin{tabular}{|c|c|c|c|c|c|c|c|c|c|c|c|}
\hline & $w_{1}$ & $w_{2}$ & $w_{3}$ & $u_{1}$ & $u_{2}$ & $u_{3}$ & $u_{4}$ & $\ldots$ & $u_{10}$ & $u_{11}$ & $u_{12}$ \\
\hline$L B$ & 5 & 1 & 3 & 2 & 3 & 4 & 0 & $\ldots$ & 0 & -20 & -20 \\
$U B$ & 7 & 4 & 7 & 7 & 10 & 6 & 20 & $\ldots$ & 20 & 20 & 20 \\
\hline
\end{tabular}

Remark 7: If $\Psi$ is linear, then $\nabla \Psi(\xi(t))$ is constant and therefore variable $\xi$ does not play any role. Then we can consider the memoryless strategy suggested in [4] for linear costs.

Remark 8: Given the average $A v[w]=\bar{w}$, the value

$$
\Psi_{\min }=\min _{B \xi=\bar{w}, \xi \in \mathcal{U}} \Psi(\xi)
$$

is a lower bound for $\Psi\left(A v\left[u_{G}\right]\right)$. To see this, note that $A v\left[u_{G}\right]$ always satisfies $B A v\left[u_{G}\right]=\bar{w}$ as well as $A v\left[u_{G}\right] \in \mathcal{U}$, and therefore $A v\left[u_{G}\right]$ is a generic feasible solution of problem (33). Also, by exploiting the definition of achievable flows in [5], we can say that if the minimizer of (33) is an achievable flow, then the lower bound $\Psi_{\min }$ is tight in the sense that $\Psi_{\min }=\Psi\left(A v\left[u_{G}\right]\right)$. Differently, if the minimizer of (33) is not an achievable flow, then $\Psi_{\text {min }}$ is not tight as $\Psi_{\min }<\Psi\left(A v\left[u_{G}\right]\right)$.

Furthermore, if we assume that $\bar{w}$ is known, then we can adopt the linear strategy $u-u_{G}=F(w-\bar{w})$ proposed [5].

Let us conclude with a brief comment on the case of network with buffers. In presence of buffers, we can achieve the same results discussed in this section. To see this, it suffices to split the strategy in two parts: the first part assuring stability and the second part deterministic optimality. Such a procedure has already been illustrated in Section IV and therefore it will not be discussed further here.

\section{NuMERICAL EXAMPLE AND SimULATIONS}

Consider again the network displayed in Fig. 1. We provide a comparison between the piecewise affine strategy (static min-max optimal strategy) $\Phi_{P W A}(w)$ defined in (13) and the gradient-based strategy (dynamic deterministically optimal strategy) $u_{G}(t)=\Phi(\xi(t), w(t))$ as in (29). In Table I we display lower and upper bounds of demand and flow.

The cost to minimize is (8) with $\rho=0.1$. Note that, we can make function $\Psi(\cdot)$ depend only on $u$ as required in Assumption 4 , by simply replacing demands $w_{1}(t), w_{2}(t)$ and $w_{3}(t)$ by three artificial flows $u_{13}(t)=w_{1}(t), u_{14}(t)=w_{2}(t)$ and $u_{15}(t)=w_{3}(t)$. Table II summarizes the optimal balancing flows $u^{(k)}$ on each vertex $w^{(k)}, k=1, \ldots, 8$. Observe that, from the network manager point of view, the cost $\Psi\left(u^{(k)}, w^{(k)}\right)$ is maximized in $w^{(5)}$. In other words, the worst demand is not the maximal demand on each node.

Now, denote $p_{k}$ the probability of being on vertex $w^{(k)}$, and consider a number of realizations with increasing probability $p_{5}$ from 0 to 1 and $p_{2}=p_{3}=p_{4}=p_{6}=p_{7}=(1-$ $\left.p_{5}\right) / 6, p_{1}=0$. Also, let $\Psi_{P W A}=\Psi\left(A v\left[\Phi_{P W A}(w)\right]\right)$ and $\Psi_{G}=\Psi\left(A v\left[u_{G}\right]\right)$ be the average costs obtained with strategies $\Phi_{P W A}(w)$ and $u_{G}(t)$, respectively. For this example, we can compute $\Psi_{P W A}(w)=\sum_{k=1}^{8} p_{k} \Psi\left(u^{(k)}, w^{(k)}\right)$ and derive $\Psi_{G}$ by simulations. In particular, we simulate a set of six realizations for $t$ from 0 to 500 , with $p_{5}=0.2,0.4,0.6,0.8,0.95$, 1 . Whichever the realization, we expect a better performance of
TABLE II

OPTIMAL FLOW $u^{(k)}$ ON EACH VERTEX $w^{(k)}, k=1, \ldots, 8$

\begin{tabular}{|c|c|c|c|c|c|c|c|c|}
\hline$k$ & 1 & 2 & 3 & 4 & 5 & 6 & 7 & 8 \\
\hline$w_{1}^{(k)}$ & 5 & 5 & 5 & 5 & 7 & 7 & 7 & 7 \\
$w_{2}^{(k)}$ & 1 & 1 & 4 & 4 & 1 & 1 & 4 & 4 \\
$w_{3}^{(k)}$ & 3 & 7 & 3 & 7 & 3 & 7 & 3 & 7 \\
\hline$u_{1}^{(k)}$ & 2 & 4.1 & 4.4 & 5.4 & 4 & 6 & 6.3 & 7 \\
$u_{2}^{(k)}$ & 3 & 3 & 3.6 & 4.6 & 3 & 3 & 3.7 & 5 \\
$u_{3}^{(k)}$ & 4 & 5.8 & 4 & 6 & 4 & 6 & 4 & 6 \\
$u_{4}^{(k)}$ & 1.7 & 2.6 & 2.5 & 2.9 & 2.9 & 3.8 & 3.7 & 4 \\
$u_{5}^{(k)}$ & 0.4 & 0.1 & 0.9 & 0.9 & 0.3 & 0 & 0.8 & 0.9 \\
$u_{6}^{(k)}$ & 2.1 & 3.7 & 2 & 3.6 & 2.1 & 3.8 & 2.0 & 3.6 \\
$u_{7}^{(k)}$ & 1.9 & 1.2 & 1.6 & 1.5 & 2.1 & 1.5 & 2 & 1.9 \\
$u_{8}^{(k)}$ & 0.3 & 1.5 & 1.8 & 2.4 & 1 & 2.1 & 2.5 & 2.9 \\
$u_{9}^{(k)}$ & 1.9 & 2.1 & 1.9 & 2.4 & 1.8 & 2.1 & 1.9 & 2.3 \\
$u_{10}^{(k)}$ & 0.6 & 1.6 & 0.9 & 2.1 & 0.5 & 1.5 & 0.8 & 2.1 \\
$u_{11}^{(k)}$ & -1.4 & -1.1 & -0.7 & -0.5 & -1.8 & -1.6 & -1.2 & -1.0 \\
$u_{12}^{(k)}$ & 0.2 & 1.5 & 0.0 & 1.2 & 0.2 & 1.6 & 0.0 & 1.2 \\
\hline$\Psi(\cdot, \cdot)$ & 18.6 & 15.6 & 8.6 & 14.4 & 20.7 & 18.9 & 12.0 & 18.5 \\
\hline
\end{tabular}

TABLE III

COST DIFFERENCE $\Psi_{P W A}-\Psi_{G}$ FOR REALIZATIONS With DifFERENT $p_{5}$. For $p_{5}=1$ (WORST REALIZATION), THE COST DifFERENCE IS NULL

\begin{tabular}{|c|c|c|c|c|c|c|}
\hline$p_{5}$ & 0.2 & 0.4 & 0.6 & 0.8 & 0.95 & 1 \\
\hline$\Psi_{P W A}-\Psi_{G}$ & 4.5 & 5.5 & 5.3 & 4.3 & 1.6 & 0 \\
\hline
\end{tabular}

(29) as evidenced in Table III, where we display the cost difference $\Psi_{P W A}-\Psi_{G}$ for different realizations (different $p_{5}$ ).

Note that in correspondence to the worst realization, characterized by $p_{5}=1$, the two strategies $\Phi_{P W A}(w)$ and $u_{G}(t)$ are equivalently optimal as $\Psi_{P W A}-\Psi_{G}=0$ (they provide the same cost $\Psi_{P W A}=\Psi_{G}=1$ ).

Furthermore, according to our expectation, we observe that $\Psi(\xi(t))$ obtained with strategy (29) always converges to $\Psi_{G}$ on the long run. This is evidenced in Fig. 4 where we plot the time evolution of the error $\Delta \Psi(t):=\Psi(\xi(t))-\Psi_{G}$ for each one of the six realizations. We can see that the error tends to zero for increasing $t$ in all of the six plots. Note the straight line in zero which is associated to the worst realization $\left(p_{5}=1\right)$. In this case, the demand is the worst one at each $t$ and $\Delta \Psi(t)=0$ which also means $\Psi(\xi(t))=\Psi_{G}=1$ for all $t$. Note that by using artificial flows $u_{13}(t), u_{14}(t)$ and $u_{15}(t)$, the variable $\xi(t)$ includes also the average demands up to time $t$.

In Fig. 5, we simulate the gradient-based strategy $u_{G}(t)$ defined in (29) (dotted) and the piece-wise strategy $\Phi_{P W A}(w)$ defined in (13) (dashed) for a realization of the demand with $p_{5}=0.4$. In particular, we plot flows $u_{i}(t)$ and demands $w_{i}(t)$ (solid) for $i=1,2,3$ from top to bottom respectively. Note that $u_{2}(t)$ obtained from the piecewise strategy (13) (dashed line, middle plot) follows the peaks of $w_{2}(t)$ (solid line, middle plot) while $u_{2}(t)$ obtained from the gradient-based strategy $u_{G}(t)$ (29) (dotted line, middle plot) does not. This is evident, for instance, in the interval from $t=10$ to $t=15$. This is due to the fact that arc 2 is over exploited for about $t=8$ where demand $w_{2}(t)=1$ and the flow in arc 2 saturates at its lower value $u_{2}(t)=3$. The gradient-based strategy $u_{G}(t)(29)$ keeps memory of the mis-match between $w_{2}(t)$ and $u_{2}(t)$ and for 


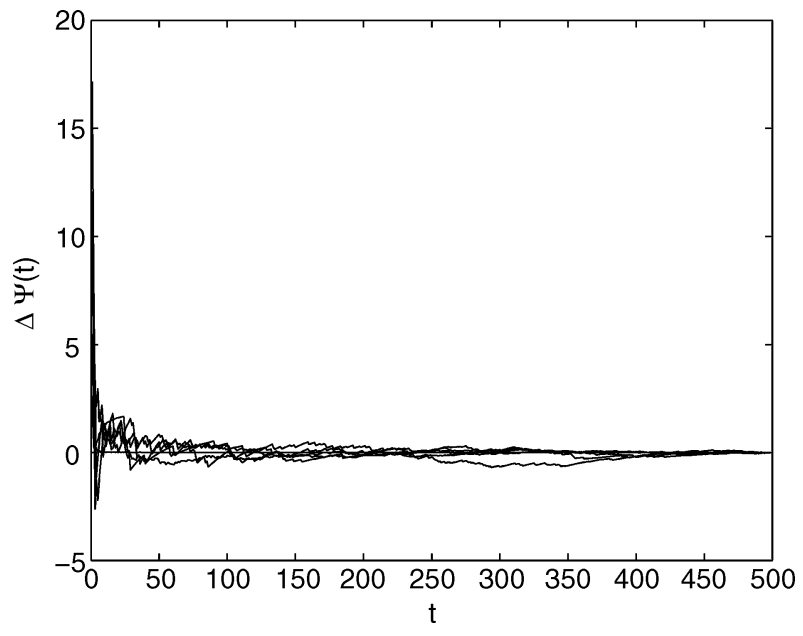

Fig. 4. Time plot of the error $\Delta \Psi(t):=\Psi(\xi(t))-\Psi_{G}$ for a set of six realizations with $p_{5}=0.2,0.4,0.6,0.8,0.95,1$. The error tends to zero for increasing $t$.
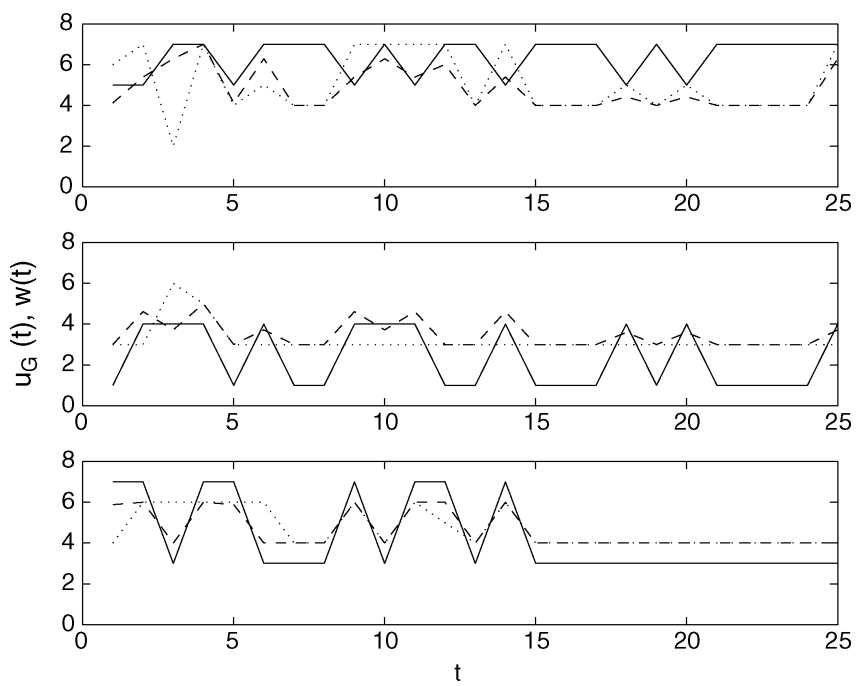

Fig. 5. Gradient-based strategy $u_{G}(t)$ (30) (dotted) and piece-wise strategy $\Phi_{P W A}(w)$ (13) (dashed) with $p_{5}=0.4$. Top: time plot of $w_{1}(t)$ (solid) and $u_{1}(t)$ obtained with the gradient-based strategy $u_{G}(t)$ (dotted) and with the piece-wise strategy $\Phi_{P W A}(w)$ (dashed); middle: time plot of $w_{2}(t)$ (solid) and $u_{2}(t)$ (dotted and dashed); bottom: time plot of $w_{3}(t)$ (solid) and $u_{3}(t)$ (dotted and dashed).

$t=10$ to $t=15$ the flow $u_{2}(t)$ (dotted line, middle) is kept constant even if the demand $w_{2}(t)$ has some peaks at its highest value.

\section{CONCLUSION}

Network flows have been dealt with under different perspectives both in robust optimization and in control theory. The present work is an attempt to emphasize connections and analogies between the two contexts.

We have studied how to robust stabilize continuous-time networks by controlling the flow with capacity constraints in the presence of demand which is unknown but bounded within a polytope. A feature of this work is that the cost is a function of the long-run average-flow and demand. We have seen that assuming for the demand a worst or a neutral behavior leads to different optimal strategies. In particular, in the first case the resulting strategy is memoryless and can be computed via convex optimization. On the contrary, in the second case we must resort to strategies with memory. We have proposed a solution based on a Lyapunov approach, in which the control is selected on-line, among the feasible flows, as the point-wise minimizer of the gradient of the cost of the average.

\section{ACKNOWLEDGMENT}

The authors wish to thank the referees for their careful reading and constructive comments.

\section{REFERENCES}

[1] E. Adida and G. Perakis, "A robust optimization approach to dynamic pricing and inventory control with no backorders," Math. Programming, ser. B, vol. 107, pp. 97-129, 2006.

[2] A. Atamturk and M. Zhang, "Two-stage robust network flow and design under demand uncertainty," Oper. Res., vol. 55, no. 4, pp. 662-673, 2007.

[3] T. Basar and G. J. Olsder, Dynamic Noncooperative Game Theory. Philadelphia, PA: SIAM, 1999.

[4] D. Bauso, F. Blanchini, and R. Pesenti, "Average flow constraints and stabilizability in uncertain production-distribution systems," in Proc. Amer. Control Conf., New York, NY, 2007, pp. 3624-3529.

[5] D. Bauso, F. Blanchini, and R. Pesenti, "Robust control policies for multi-inventory systems with average flow constraints," Automatica, vol. 42, no. 8, pp. 1255-1266, 2006.

[6] A. Ben-Tal, L. El Ghaoui, and A. Nemirovski, "Foreword: Special issue on robust optimization," Math. Programming, ser. B, vol. 107, pp. 1-3, 2006.

[7] A. Bemporad, F. Borrelli, and M. Morari, "Min-max control of constrained uncertain discrete-time linear systems," IEEE Trans. Autom. Control, vol. 48, no. 9, pp. 1600-1606, Sep. 2003.

[8] D. P. Bertsekas and I. B. Rhodes, "On the minimax reachability of target set and target tubes," Automatica, vol. 7, pp. 233-247, 1971.

[9] D. P. Bertsekas, "Linear convex stochastic control problems over an infinite horizon," IEEE Trans. Autom. Control, vol. AC-18, no. 3, pp. 314-315, Jun. 1973.

[10] D. Bertsimas and A. Thiele, "A robust optimization approach to inventory theory," Oper. Res., vol. 54, no. 1, pp. 150-168, 2006.

[11] D. Bertsimas and C. Caramanis, "Finite Adaptability in Multistage Linear Optimization," IEEE Trans. Autom. Control, to be published.

[12] F. Blanchini and S. Miani, Set-Theoretic Methods in Control. Boston, MA: Birkhäuser, 2008.

[13] F. Blanchini, S. Miani, and F. Rinaldi, "Guaranteed cost control for multi-inventory systems with uncertain demand," Automatica, vol. 40, no. 2, pp. 213-224, 2004.

[14] F. Blanchini, S. Miani, and W. Ukovich, "Control of production-distribution systems with unknown inputs and system failures," IEEE Trans. Autom. Control, vol. 45, no. 6, pp. 1072-1081, Jun. 2000.

[15] F. Blanchini, F. Rinaldi, and W. Ukovich, "A network design problem for a distribution system with uncertain demand," SIAM J. Optim., vol. 7, pp. 560-578, 1997.

[16] F. Blanchini, F. Rinaldi, and W. Ukovich, "Least inventory control of multi-storage systems with non-stochastic unknown input," IEEE Trans. Robot. Autom., vol. 13, no. 5, pp. 633-645, Oct. 1997.

[17] E. K. Boukas, H. Yang, and Q. Zhang, "Minimax production planning in failure-prone manufacturing systems," J. Optim. Theory Appl., vol. 82, no. 2, pp. 269-286, 1995.

[18] A. Erera, J. Morales, and M. W. P. Savelsbergh, "Robust optimization for empty repositioning problems," Oper. Res., vol. 57, no. 2, pp. 468-483, Mar./Apr. 2009.

[19] E. Khmelnitsky and M. Tzur, "Parallelism of continuous- and discretetime production planning problems," IIE Trans., vol. 36, pp. 611-628, 2004.

[20] B. Grunbaum, Convex Polytopes. New York: Wiley, 1967.

[21] O. Kostyukova and E. Kostina, "Robust optimal feedback for terminal linear-quadratic control problems under disturbances," Math. Programming, ser. B, vol. 107, pp. 131-153, 2006.

[22] D. Luenberger, Optimization via Vector Spaces Methods. New York: Wiley, 1997. 
[23] S. Mudchanatongsuk, F. Ordóñez, and J. Liu, "Robust solutions for network design under transportation cost and demand uncertainty," $J$. Oper. Res. Soc., vol. 59, pp. 652-662, 2008.

[24] F. Ordóñez and J. Zhao, "Robust capacity expansion of network flow," Network, vol. 38, pp. 136-145, 2007.

[25] E. A. Silver and R. Peterson, Decision System for Inventory Management and Production Planning. New York: Wiley, 1985.

[26] S. Sethi, H. Zhang, and Q. Zang, Average Cost Control of Stochastic Manufacturing Systems. New York: Springer, 2004.

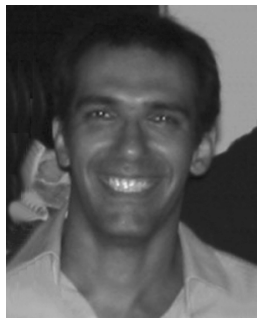

Dario Bauso was born in Palermo, Italy, on May 17, 1974. He received the Laurea degree in aeronautical engineering and the Ph.D. degree in automatic control and system theory from the University of Palermo, Palermo, Italy, in 2000 and 2004, respectively.

From October 2001 to June 2002, he was a Visiting Scholar at the Mechanical and Aerospace Engineering Department, University of California, Los Angeles. Since 2005, he has been Assistant Professor of Operations Research with the Engineering Faculty, University of Palermo. He is affiliated with the Department of Computer Science of the same University. His research interests are in the field of optimization problems, manufacturing systems, inventory control, optimal and distributed control, and game theory.

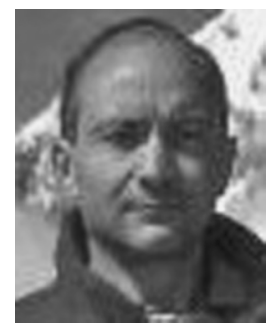

Franco Blanchini was born in Legnano, MI, on December 29, 1959.

$\mathrm{He}$ is Professor at the Engineering Faculty of the University of Udine where he teaches dynamic system theory and automatic control. He is a member of the Department of Mathematics and Computer Science of the same university and he is Director of the System Dynamics Laboratory. He has been Associate Editor of Automatica from 1996 to 2006.

Dr. Blanchini received the 2001 ASME Oil \& Gas Application Committee Best Paper Award as a co-author of the article "Experimental evaluation of a High-Gain Control for Compressor Surge Instability," the 2002 IFAC prize survey paper award as author or the article "Set Invariance in Control—a survey", Automatica, November, 1999, and the award Automatica Certificate of Outstanding Service. He was a member of the Program Committee of the 36th, 38th, 40th and 42th IEEE Conferences on Decision and Control. He was Chairman of the 2002 IFAC workshop on Robust Control, Cascais, Portugal. He has been Program Vice-Chairman for the conference Joint CDC-ECC, Seville, Spain, December 2005. He was Program Vice-Chairman for Tutorial Sessions for the 2088 IEEE Conference on Decision and Control, Cancun, Mexico.

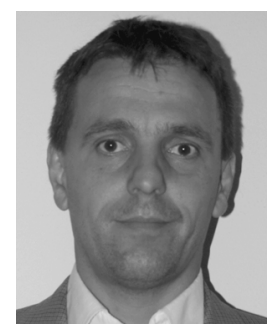

Raffaele Pesenti is Professor of Operations Research at the University of Venice, Venice, Italy. His works appear in journals such as Automatica, Bioinformatics, the IEEE TRANSACTIONS ON AUTOMATIC CONTROL, the IEEE TRANSACTIONS ON ROBOTICS AND AutOMATION, NeTworks, the SIAM Journal on Control and Optimization, and the SIAM Journal on Optimization, Transportation Science. His major area of research are: management and evaluation of complex systems with application in logistics and biology. The interests in this field are devoted to the study of the strategic and analytical elements of the systems design and to the development of models and methods which may find application in the day by day operations. 